\title{
WHITTAKER'S CON'TRIBUTIONS TO THE THEORY OF RELATIVITY
}

\author{
by J. L. SYNGE
}

TEN papers deal with the theory of relativity, and all are concerned in some way with electromagnetism. Those who know Whittaker through his Modern Analysis and Analytical Dynamics will recognise in these papers the same mastery over complicated situations which enabled him to disdain the support of notational refinements, that same elegance, brevity and persuasive charm which make difficult arguments seem easier than they really are. The new element which emerges is the strongly geometrical approach; but he remains true to the Lagrange tradition and draws no diagrams of space-time, although these must surely have been before his mind's eye and would have helped his readers.

There are certain affiliations between these ten papers, and they might be arranged in groups; but it is simpler to deal with them in chronological order. In this report, the summation convention is understood, the permutation symbol $\epsilon_{r s m n}$ is used, partial differentiation is indicated by a comma $\left(X_{r, \varepsilon}\right)$ and covariant differentiation by a vertical stroke $\left(X_{r \mid s}\right)$. These notational devices, now in common use, were not employed by Whittaker.

(a) "On tubes of electromagnetic force," Proc. Roy. Soc. Edin., 42 (1921), $1-23$.

"The object of the present paper is to introduce certain surfaces, which will be shown to play the same part in the general electromagnetic field as Faraday's tubes of force do in electrostatic and magnetostatic fields."

The fabric of this paper is woven out of Maxwell's equations in vacuo, and one other thing-the condition

$$
d_{x} h_{x}+d_{y} h_{y}+d_{z} h_{z}=0
$$

expressing the orthogonality of the electric and magnetic vectors $(d, h)$. This condition is Lorentz-invariant. In the last pages of the paper this restriction is lifted and the generalization indicated, but the results are then more complicated and less interesting, and throughout this account of the paper (1) will be assumed as a restriction on the field under consideration.

To express the essentials of the work compactly, let us use Minkowskian coordinates with $x_{4}=i c t$ and the electromagnetic tensor $F_{r s}$, where

$$
\begin{array}{lll}
d_{x}=i F_{14}, & d_{y}=i F_{24}, & d_{z}=i F_{34}, \\
h_{x}=F_{23}, & h_{y}=F_{31}, & h_{z}=F_{12} .
\end{array}
$$

We need also the dual $F^{*}{ }_{r s}=\frac{1}{2} i \epsilon_{r s m n} F_{m n}$. Then Maxwell's equations may be written

$$
F_{r s, s}=0, F_{r s, s}^{*}=0 \text {. }
$$

The condition ( 1 ) is equivalent to

$$
\operatorname{det} F_{r s}=0 \text { or } F_{23} F_{14}+F_{31} F_{24}+F_{12} F_{34}=0 .
$$


Consider now the total differential equations

$$
F_{r s} d x_{s}=0 \text {, }
$$

which are in general inconsistent, but which, in view of (4), are equivalent to only two equations. These equations, and the fact that they are integrable, form the core of the paper. They are integrable, in the sense that they possess integrals $\phi=$ const., $\psi=$ const., by virtue of Maxwell's equations (3) and the condition (4), $\phi$ and $\psi$ being solutions of the following partial differential equations adjoint to the total differential equations (5) :

$$
F_{r s}^{*} f_{, s}=0 \text {. }
$$

Thus from (5) we get $\infty^{2} 2$-spaces, which Whittaker called electropotential surfaces. They have the property that the curves of intersection of these surfaces with $t=$ const. are the magnetic lines of force, and in an electrostatic field these surfaces are the ordinary equipotential surfaces.

The total differential equations

$$
F_{r s}^{*} d x_{s}=0
$$

likewise give $\infty^{2} 2$-spaces-these are Whittaker's magnetopotential surfaces.

At each event the electropotential surface is absolutely orthogonal to the magnetopotential surface, absolute orthogonality being Whittaker's expression to indicate that every vector in one 2-space is orthogonal (in the Minkowskian sense) to every vector in the other.

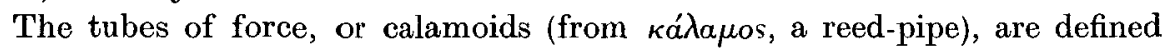
in terms of electropotential and magnetopotential surfaces. We pick out 3 -spaces $G$ and $H, G$ being formed from $\infty^{1}$ electropotential surfaces and $H$ from $\infty^{1}$ magnetopotential surfaces. The intersection $\Sigma$ of $G$ and $H$ is a calamoid ; a calamoid is a 2-space, and there are $\infty^{2}$ of them altogether. In a purely electrostatic field, or in a purely magnetostatic field, the calamoids become the ordinary Faraday tubes of force.

Whittaker gives explicitly the partial differential equations of the calamoids. In tensor notation, they read

where

$$
F_{r s} X_{r s}=0, F_{r s}^{*} X_{r s}=0
$$

$$
X_{r s}=\frac{\partial\left(x_{r}, x_{s}\right)}{\partial(u, v)}
$$

He uses these partial differential equations to express Maxwell's equations in integral form: If $S$ be any closed 2-space to which we can fit an open 3 -space not containing electrons, then

$$
\iint F_{r s} X_{r s} d u d v=0, \quad \iint F_{r s}^{*} X_{r s} d u d v=0,
$$

the integrals being taken over $S$.

(b) "On Hilbert's world-function," Proc. Roy. Soc. A, 113 (1927), 496-511. 
This is a difficult paper, physically and mathematically. Whittaker did not take kindly to the ideas of quantum theory, and thought that light quanta should be explained within Maxwellian theory, enlarged by the inclusion of magnetic currents. The character of these currents is not made clear by the statement that if " we give the name "magnetism' to a non-circuital character. of the magnetic lines of force, then we may say that magnetic currents consist in the motion of ' magnetism' but we should not attribute to 'magnetism' a substantiality like that of matter."

It is, of course, easy to include magnetic currents in the Maxwellian scheme. All we have to do is to replace the zeros on the right-hand sides of four of the Maxwellian equations with the components of magnetic 4-current. When this modification has been made, then, Whittaker wrote, "we can prove the existence of finite light-pulses or light-quanta, i.e., we can construct solutions of the equations which at every instant are null everywhere outside certain finite regions, and which are propagated, without change of size or shape, with the velocity of light. We can moreover reconcile the classical theory, thus extended, with the theorem which Einstein inferred some years ago from thermodynamic considerations, that a pulse of light which is emitted by one atom is ultimately all gathered together again on another single atom."

This is a surprising statement, as to the validity of which one may entertain some scepticism. Although this idea lay behind the paper under consideration, it is not enlarged on sufficiently for one to form a more definite idea of the electromagnetic field which Whittaker had in mind, and it is a pity that he did not explain it further within the simpler framework of special relativity.

Mathematically, the paper is of considerable complexity, and I have not been able to get to the heart of the matter. We have before us a certain physical system ; it consists of material particles in incoherent motion, carrying electric and magnetic charges (in spite of what has been quoted above). These particles move in a combined gravitational and electromagnetic field.

We seek a set of differential equations which will describe this situation. If we leave out the magnetic charges, the situation is a familiar one, and we know the equations which seem appropriate. They consist of the Einstein field equations, having on the right-hand side an energy tensor made up of a contribution from the matter and a contribution from the electromagnetic field, both well known in form. We should also write down the form of Maxwell's equations suited to general relativity. From these field equations we can derive (through the vanishing of the divergence of the Einstein tensor) the equations of motion of matter-they are equivalent to the Lorentz law of ponderomotive force. If we now put in the magnetic charges, the only modification required is in Maxwell's equations (to allow for magnetic 4-current).

Thus, if our object is merely to obtain a reasonable set of equations governing the situation described above, that object is easily attained, and indeed the equations outlined above are those which Whittaker finally obtains. 
But the whole point of the paper is the deduction of these equations from a variational principle of the form

$$
\delta \int H \sqrt{ }(-g) d x^{0} d x^{1} d x^{2} d x^{3}=0
$$

and the problem is to choose a suitable function $H$ and prescribe a suitable way of carrying out the variation. Here the inclusion of the magnetic current does make a significant difference. If magnetic currents are absent, those four Maxwellian equations which have zero on the right-hand side imply the existence of a 4-potential, and that 4-potential (multiplied by the 4-current) appears in $H$. But when magnetic currents are present, this procedure breaks down, and Whittaker's problem was to introduce a 4-potential in some indirect way. This he succeeded in doing, but the argument is not at all clear to me, and I cannot attempt to describe it in brief compass.

(c) "On electric phenomena in a gravitational field," Proc. Roy. Soc. A, 116 (1927), 720-735.

Although an electromagnetic field influences a gravitational field through its energy tensor, this effect is in general small, and in this paper Whittaker studies electromagnetic fields superimposed on a space-time which has an assigned geometry consistent with Einstein's field equations in vacuo.

One half of Maxwell's equations (there is no question of a magnetic current here) ensure the existence of the electromagnetic potential-vector $\phi_{p}$, and when the condition

$$
g^{p q} \phi_{p \mid q}=0
$$

is imposed on $\phi_{p}$, the other half of Maxwell's equations require that $\phi_{p}$ should satisfy

$$
g^{q r} \phi_{p_{1} r q}+K_{p}^{8} \phi_{s}=j_{p}
$$

where $K_{p}^{8}$ is the mixed form of the contracted Riemann tensor and $j_{p}$ is the 4-current. Since the field equations of empty space-time are to be used, we put $K_{p}^{8}=0$, and so we have to deal with the set of linear partial differential equations comprised in (1) and

$$
g^{q r} \phi_{p \mid r q}=j_{p}
$$

After these general preliminaries, Whittaker confines himself to two gravitational fields, namely, the Schwarzschild spherically symmetric field with

and a field with

$$
d s^{2}=\left(1-\frac{a}{r}\right) d t^{2}-\frac{1}{c^{2}}\left(\frac{d r^{2}}{1-\frac{a}{r}}+r^{2} d \theta^{2}+r^{2} \sin ^{2} \theta d \phi^{2}\right),
$$

$$
d s^{2}=\left(1+\frac{2 g x}{c^{2}}\right) d t^{2}-\frac{1}{c^{2}}\left(\frac{d x^{2}}{1+\frac{2 g x}{c^{2}}}+d y^{2}+d z^{2}\right),
$$

where $g$ is a constant. This last field he calls quasi-uniform and remarks that 
it is the simplest of all types of gravitational field ; it is obtained from (4) by a limiting process in which the gravitating centre is removed to infinity and its mass increased appropriately. But unfortunately he overlooked the fact (recently pointed out to me by Dr F. A. E. Pirani) that (5) is actually a flat line-element; it reduces to

if we put

$$
d s^{2}=d \tau^{2}-d \xi^{2}-d \eta^{2}-d \zeta^{2}
$$

$$
\begin{gathered}
\tau=\frac{c}{g} \sqrt{1+\frac{2 g x}{c^{2}}} \sinh \frac{g t}{c}, \xi=\frac{c}{g} \sqrt{1+\frac{2 g x}{c^{2}}} \cosh \frac{g t}{c}, \\
\eta=\frac{y}{c}, \quad \zeta=\frac{z}{c} .
\end{gathered}
$$

Thus formulæ based on (5) are not formulæ for a gravitational field but for curvilinear coordinates $(x, y, z, t)$ in a flat space-time; as such, they have a certain interest, although it is not the interest they were supposed to have.

Dealing first with this quasi-uniform field, Whittaker writes out the equations (1) and (3) explicitly, and notes that for electrostatic problems the essential equation is

$$
\left(1+\frac{2 g x}{c^{2}}\right) \frac{\partial^{2} \phi_{0}}{\partial x^{2}}+\frac{\partial^{2} \phi_{0}}{\partial y^{2}}+\frac{\partial^{2} \phi_{0}}{\partial z^{2}}=0
$$

which generalizes Laplace's equation and reduces to it when $g=0$. Several analogues of harmonic functions are obtained. First, the analogue of

is

$$
e^{-k x} J_{m}(k \rho) \underset{\sin }{\cos } m \phi
$$

$$
\phi_{0}=\left(1+\frac{2 g x}{c^{2}}\right)^{\frac{1}{2}} J_{1}\left\{\frac{i k c^{2}}{g}\left(1+\frac{2 g x}{c^{2}}\right)^{\frac{1}{2}}\right\} J_{m}(k \rho){ }_{\sin }^{\cos m \phi . ~}
$$

Secondly, the analogue of a constant is the rational function

$$
\phi_{0}=\frac{c^{2}+2 g x}{\left\{c^{4}+2 c^{2} g x+g^{2}\left(y^{2}+z^{2}\right)\right\}^{2}} .
$$

Thirdly, the analogue of $1 / r$ is

$$
\phi_{0}=\frac{1+\frac{g x}{c^{2}}+\frac{g^{2}}{2 c^{4}}\left(y^{2}+z^{2}\right)}{\left\{x^{2}+y^{2}+z^{2}+\frac{g}{c^{2}} x\left(y^{2}+z^{2}\right)+\frac{g^{2}}{4 c^{4}}\left(y^{2}+z^{2}\right)^{2}\right\}^{\frac{1}{2}}},
$$

representing the potential of a unit point-charge at the origin. This is generalized to yield the potential due to unit point-charge situated at an arbitrary point, and hence he obtains an integral representing the most general electrostatic potential in space-time with metric as in (5).

Turning to the Schwarzschild field (4), he obtains the analogue of Laplace's equation, separates the variables, and gets the solution

$$
\phi_{0}=\frac{2 .(n-1) ! n !}{(2 n) !} a^{n-1}(r-a) \frac{d P_{n}(z)}{d z} P_{n}^{m}(\cos \theta) \stackrel{\cos }{\sin } m \phi
$$


where $z$ is defined by $r=\frac{1}{2} \alpha(z+1)$. This is the analogue of the harmonic function

$$
r^{n} P_{n}^{m}(\cos \theta){ }_{\sin }^{\cos } m \phi,
$$

and, like it, may be used to construct solutions of electrostatic problems in the field of a single gravitating centre. In fact, he uses it to construct, in the form of a double integral, the electrostatic field of a point-charge placed in such a gravitational field.

The paper concludes with a study of electromagnetic waves in the Schwarzschild gravitational field. With no 4-current, and with $\phi_{0}=\phi_{1}=\phi_{2}=0$, there is just one equation to satisfy-a second-order linear partial differential equation for $\phi_{3}$. The variables are separated, and a solution obtained in the form

$$
\phi_{3}=e^{i p t} f(r) \sin ^{2} \theta \frac{d P_{n}(\cos \theta)}{d(\cos \theta)}
$$

where $f(r)$ satisfies

$$
\frac{r-a}{r} \frac{d^{2} f}{d r^{2}}+\frac{a}{r^{2}} \frac{d f}{d r}+\left\{\frac{p^{2} r}{r-a}-\frac{n(n+1)}{r^{2}}\right\} f=0,
$$

which is a linear differential equation of the second order with three singular points, namely, $0, a$ and $\infty$. The exponents at $r=0$ are 0 and 2 , and the exponents at $r=\alpha$ are $i p a / c$ and $-i p a / c$, while $r=\infty$ is an irregular singular point at which $f$ behaves like

$$
e^{ \pm i p r / c} r \pm i a p / c .
$$

Whittaker notes that this equation is akin to Mathieu's equation, but not identical with it. For high frequencies, he gives the approximate solution

$$
f(r)=A e^{i p r / c}(r-\alpha)^{i p a / c}+B e^{-i p r / c}(r-\alpha)^{-i p a / c} \text {, }
$$

and, for arbitrary frequencies, he shows how to develop $f$ in negative powers of $p$.

(d) "Note on the law that light-rays are the null geodesics in a gravitational field," Proc. Cambridge Phil. Soc., 24 (1928), 32-34.

Whittaker seeks to rationalize, on the basis of Maxwell's equations, Einstein's assumption that the tracks of light-rays are null geodesics in a gravitational field. He regards the metric tensor $g_{p q}$ as given, and notes that the electromagnetic potential-vector satisfies partial differential equations of the type

$$
g^{p q} \frac{\partial^{2} \phi_{r}}{\partial x^{p} \partial x^{q}}+\ldots=0
$$

the term written explicitly being the only one which involves second derivatives of the $\phi_{r}$ 's. A characteristic being defined as a manifold on which the existence theorem of P.D.E.'s breaks down, a characteristic of (1) is a hypersurface such that a solution is not uniquely defined by the values on it of the $\phi_{r}$ 's and their first derivatives. From the known theory of characteristics it follows 
that the characteristic hypersurfaces $f\left(x^{0}, x^{1}, x^{2}, x^{3}\right)=0$ of the partial differential equations ( 1 ) are the solutions of the partial differential equation

$$
g^{p q} \frac{\partial f}{\partial x^{p}} \frac{\partial f}{\partial x^{q}}=0
$$

It follows further from the known theory of characteristics that the characteristics of (2) are curves, and that these characteristic curves are the null geodesics for the metric $d s^{2}=g_{p q} d x^{p} d x^{q}$.

If, then, an electromagnetic disturbance is originally confined to a limited region of space, and the history of the bounding surface of the disturbance is represented by the equation $f\left(x^{0}, x^{1}, x^{2}, x^{3}\right)=0$, the function $f$ satisfies (2), and the hypersurface with this equation is an aggregate of null geodesics.

The paper concludes as follows:

"But when we speak of a 'ray of light', we mean a disturbance which is filiform in space-time (or, in three-dimensional space, a disturbance which is localised at any instant in an infinitely small region, varying from instant to instant). Clearly if this filiform region is to be composed of the abovementioned characteristic curves, it can consist of only one of them : that is to say, a ray of light in a gravitational field is a null geodesic of space-time, which is the theorem to be proved."

It is, I think, a pity that Whittaker confused the mathematical issue by this statement. But it was no afterthought, for the Introduction makes it clear that this was his goal and that he thought he had attained it. But I don't think that he did, or that anyone else could, because the goal does not exist. As I see the situation, it seems that we are faced with two irreconcilable ways of looking at electromagnetic phenomena: on the one hand we have the light-quantum or photon, and, on the other hand, Maxwellian field theory. But at first we are reluctant to accept their irreconcilability. We try to obtain a Maxwellian field corresponding to a concentration of electromagnetic disturbance travelling with the speed of light. According to a previous paper, $(b)$ above, Whittaker seems to have thought that the inclusion of magnetic currents would overcome the difficulty of constructing such electromagnetic concentrations, but he gave no indication how it was to be done. In the paper under consideration there is no question of magnetic currents, and indeed Whittaker points out in the Introduction that any " ray" does in fact spread out. Thus the filiform disturbance is a pure dream-child, a non-existent dream-child, and any statements made about it have no meaningful content.

This paper is of fundamental importance in connection with the mathematical structure of Maxwellian fields, but it does not remove the mystery surrounding the physical role played by the null geodesic in the propagation of light.

We may pause for a moment, with this short paper before us, to ask a question which might (and should) be asked about any man who has made a deep impression on the age in which he lived. What passion drove him on? I mean the real passion, sought beyond the conventional answers suited 
to the naïve, but tremendously active, period of science in which he matured. I don't think that Whittaker was impelled by a passion to find out how nature really works. That is, I know, a frightfully heterodox thing to say of a great scientist, but $I$ think it is true, and explains in some measure his denigration (to use a word which is a little too strong) of Einstein in relation to the foundation of the theory of relativity. For Einstein, who had only a small fraction of Whittaker's mathematical skill, was in his early days impassioned to know the real nature of things, with a success which everyone knows.

I see Whittaker as a consummate artist in the formal symbolism of mathematics, his passion being to make the symbols dance to his tune and to take their place in persuasive arguments of great economy, inessential details being suppressed. But this passion alone did not suffice, for he yearned on the one hand towards that precision of mathematical thought which was lacking among the British mathematicians of his age (a situation which he did much to remedy), and on the other hand he yearned for contact with the realities of the physical universe-realities which, in their broader aspects, he could treat with philosophical depth, but which he was not adapted to uncover for himself. He could narrate in a most lucid way the history of electromagnetism, both mathematically and physically, but the passion for narration and the passion for creation are very different. He had, it seems, no consuming passion for physical reality, no more than Lagrange or Hamilton had; he was more akin to them intellectually, than to Newton or Maxwell or Kelvin or Rayleigh.

(e) "The influence of gravitation on electromagnetic phenomena," Journ. Lond. Math. Soc., 3 (1928), 137-144.

This was a lecture, and in it Whittaker refers to some of the results given in (c) above, now comparing electromagnetic theory in a gravitational field to Maxwellian theory in a medium whose specific inductive capacity and magnetic permeability vary from point to point, but noting that the resemblance is not quite perfect. He also discusses null geodesies in the Schwarzschild field with metric as in (4) of $\S(c)$, noting that the null geodesics in the plane $\theta+\pi / 2$ satisfy the differential equation

$$
\left(\frac{d U}{d \phi}\right)^{2}=a U^{3}-U^{2}+k^{2}
$$

where $U=1 / r$ and $k$ is a constant, and hence are given in terms of the Weierstrass elliptic function by

$$
\frac{1}{r}=\frac{1}{3 a}+\frac{4}{a} \wp \rho(\phi+C) .
$$

If $k$ has the particular value $2 /\left(3^{3 / 2} a\right)$, we get solutions in terms of hyperbolic functions, namely,

and

$$
\frac{a}{r}=\frac{2}{3}+\frac{1}{\sinh ^{2}\left(\frac{1}{2} \phi+C\right)}
$$

$$
\frac{a}{r}=\frac{2}{3}-\frac{1}{\cosh ^{2}\left(\frac{1}{2} \phi+C\right)} \text {. }
$$


"These rays are spirally asymptotic to the circle $r=\frac{3}{2} a$; the second equation represents light which, coming from infinity towards the mass, is ' captured ' by it, and never gets away again, but circles round it for ever; the first equation, on the other hand, represents luminous energy which is, and always has been, imprisoned in the immediate neighbourhood of the mass, since $r$ is less than $\frac{3}{2} a$ for all values of $\phi . "$

"The capture and imprisonment of radiation by the intense gravitational field surrounding a point-mass is a remarkable theoretical possibility, markedly different from anything in pre-relativity physics."

$(f)$ " On the potential of electromagnetic phenomena in a gravitational field," Proc. Roy. Soc. A, 120 (1928), 1-13.

This paper follows the same general pattern as $(c)$ and $(e)$-the exploration of the changes in electromagnetic theory consequent on the curvature of spacetime. The starting-point is the well-known Liénard-Wiechert retarded potential, which Whittaker writes in the form

$$
\left(\phi_{0}, \phi_{1}, \phi_{2}, \phi_{3}\right)=\left(e / s,-e v_{x} / s,-e v_{y} / s,-e v_{z} / s\right),
$$

where

$$
s=\bar{r}+\left\{\left(\bar{x}^{\prime}-x\right) v_{x}+\left(\bar{y}^{\prime}-y\right) v_{y}+\left(\bar{z}^{\prime}-z\right) v_{z}\right\} / c .
$$

So far space-time is flat; $E(x, y, z, t)$ is the event where the observation of the field is made; $\bar{E}\left(\bar{x}^{\prime}, \bar{y}^{\prime}, \bar{z}^{\prime}, \bar{t}\right)$ is the event where the world-line of the electron cuts the null-cone drawn into the past from $E ; \vec{r}$ is the distance between $(x, y, z)$ and $\left(x^{\prime}, y^{\prime}, z^{\prime}\right)$; and $\left(v_{x}, v_{y}, v_{z}\right)$ is the velocity of the electron at $\bar{E}$.

Still supposing space-time to be flat, Whittaker seeks an expression for $\phi_{p}$ valid, not only for Galilean coordinates as in (1), but for all curvilinear coordinates in space-time. To this end, he writes down the formula

$$
\phi_{p}=\frac{e}{c} g^{a r} \sigma_{p \mid q r}, \quad \sigma_{p}=\tau_{\mid p} / g^{l m} \tau_{\mid l m} .
$$

Here $\tau$ is the proper time of the electron at the event $\bar{E}$ considered above. Obviously, this formula is covariant, and therefore valid in general if valid for Galilean coordinates. By direct calculation, he shows that (3) reduces to (1) for Galilean coordinates, and so (before accosting the curvature of space-time) he has in (3) an elegant covariant expression for the retarded 4-potential.

What happens when space-time is curved ? Is (3) still valid? Whittaker points out that $\phi_{p}$ must be given by some covariant formula involving nothing but the fundamental tensor $g_{p q}$ and the covariant derivatives of the proper time $\tau$; however, the combination may not be as in (3). To test a particular case, he applies (3) to the "quasi-uniform field" (5) of (c), and verifies that it yields $\phi_{0}$ as in (9) of (c) for a charge at rest at the origin. Actually, this is no test of the validity of (3) in curved space-time, since, as we have seen, (5) of (c) is flat. 
At the end of the paper, he seeks to establish a result which would be remarkable, if true. The invariant proper time $\tau$ is determined by the world. line of the electron and the coordinates $x^{p}$ of the event $E$ at which the observation is made. When we calculate the first order partial derivatives of $\tau$, we find that they depend, not only on the values of $x^{p}$, but also on the tangent to the world-line at $\bar{E}$, or, in other words, on the velocity of the electron. Similarly, the covariant derivatives of the second order, $\tau_{\mid l m}$, involve both the velocity and acceleration of the electron. The result which Whittaker states is this : the combination $\sigma_{p}$ of (3) involves neither the velocity nor the acceleration of the electron.

The argument begins by assigning coordinates $\chi^{p}$ to $\bar{E}$ and writing

$$
\Omega(\chi, x)=0
$$

for the null-cone belonging to $\chi^{p}$. Then $\Omega$ satisfies

$$
g^{l m} \frac{\partial \Omega}{\partial x^{l}} \frac{\partial \Omega}{\partial x^{m}}=0
$$

Whittaker differentiates with respect to $\chi^{r}$, obtaining

$$
g^{l m} \frac{\partial \Omega}{\partial x^{l}} \frac{\partial^{2} \Omega}{\partial x^{m} \partial \chi^{r}}=0
$$

an equation which is essential to the argument, but which is incorrect, as we may see as follows. Take flat space-time with $g_{p q}$ constant. Then the equation of the null-cone is

and we get

$$
\Omega(\chi, x)=\frac{1}{2} g_{p q}\left(x^{p}-\chi^{p}\right)\left(x^{q}-\chi^{q}\right)=0,
$$

$$
\frac{\partial \Omega}{\partial x^{m}}=g_{m p}\left(x^{p}-\chi^{p}\right), \frac{\partial^{2} \Omega}{\partial x^{m} \partial \chi^{r}}=-g_{m r}, \quad g^{l m} \frac{\partial \Omega}{\partial x^{l}} \frac{\partial^{2} \Omega}{\partial x^{m} \partial \chi^{r}}=-g_{r p}\left(x^{p}-\chi^{p}\right) \ldots
$$

These last four expressions do not vanish unless $x^{p}=\chi^{p}$, which of course is not the case. The fact that space-time is actually curved could not validate (6), which we have just seen to be false for flat space-time. The error came from overlooking the fact that (5) is not true for all values of the eight arguments in $\Omega$, but only for those values which satisfy $\Omega=0$.

$(g)$ "On the definition of distance in curved space, and the displacement of the spectral lines of distant sources," Proc. Roy. Soc. A, 133 (1931), 93-105.

Newtonian concepts die hard, and astronomers often speak of the distances and velocities of recession of nebulæ as though the concepts of distance and velocity required no redefinition in terms suited to the general theory of relativity. Into this philosophical obscurity, Whittaker shoots a deft ray of light.

"When the astronomer asserts that "the distance of the Andromeda nebula is a million light-years,' he is stating a relation between the worldpoint occupied by ourselves at the present instant and the world-point occupied by the Andromeda nebula at the instant when the light left it which arrives here now ; that is, he is asserting a relation between two world-points such 
that a light-pulse, emitted by one, arrives at the other; or in geometrical language, between two world-points which lie on the same null geodesic. The spatial distance of two material particles in a general Riemannian space-time may, then, be thought of as a relation between two world-points which are on the same null geodesic."

His definition of spatial distance is not only mathematically elegant; it is practical because it is connected with the way in which astronomers actually compute the distance of a very remote object, namely, by comparing its observed apparent brightness with its assumed absolute brightness. Consider a thin pencil of null geodesics (rays of light) issuing from a star at an event $A$, and passing through and close to an event $B$ in the history of an observer. Then the distance from $A$ to $B$ is defined to be proportional to the square root of the two-dimensional area in which this thin null pencil cuts the observer's instantaneous space (the 3-space orthogonal to his world-line). This definition makes the distance from $A$ to $B$ depend on the direction of the observer's world-line at $B$ (i.e., on the motion of the observer), and Whittaker remarks that it is quite proper that it should so depend.

This simple definition of spatial distance is illustrated by applying it to the de Sitter world. The Doppler effect is also discussed, the argument being based on the fact that if $\tau_{0}$ denotes the proper-time of the observer at the instant when he receives a light-signal which left the star at the star's propertime $\tau$, then the Doppler effect $\delta \lambda / \lambda$ (where $\lambda$ denotes wave-length) is given by the simple equation

$$
\frac{\delta \lambda}{\lambda}=\frac{d \tau_{0}}{d \tau}-1
$$

Letting the radius of the de Sitter world tend to infinity, he shows that his general formula for the Doppler effect reduces to the well-known formula of special relativity.

(h) (with W. O. Kermack and W. H. McCrea). " On properties of null geodesics, and their application to the theory of radiation," Proc. Roy. Soc. Edin., 53 (1933), 31-47.

As in $(g)$, questions connected with spatial distance and the Doppler effect are treated in terms of null geodesics, but the work proceeds on more general and rather different lines. The basic ideas were in the air, and acknowledgments are made of overlaps with other writers.

First, in a $V_{n}$ with indefinite metric, the properties of null geodesics are discussed. An ordinary geodesic has an intrinsic parameter (the separation $s$ ) ; a null geodesic $\Gamma$ has no such unique parameter, but something nearly as good-an intrinsic parameter $\lambda$ in terms of which the equations of $\Gamma$ read

$$
\frac{d^{2} x^{p}}{d \lambda^{2}}+\left\{\begin{array}{c}
p \\
m n
\end{array}\right\} \frac{d x^{m}}{d \lambda} \frac{d x^{n}}{d \lambda}=0
$$

and which can be submitted only to a linear transformation without destroying this property. This group of parameters can also be reached by assigning a E.M.S.-D 
tangent vector $\eta^{p}$ at some point $C$ of $\Gamma$, subjecting $\eta^{p}$ to parallel transport on $\Gamma$, and defining $\lambda$ by $\eta^{p}=d x^{p} / d \lambda$ at $C$. Such a null vector $\eta^{p}$ is called a transport vector. This special parameter $\lambda$ (with a normalising condition at some one point) is used to define spatial distance on a null geodesic.

The scene now shifts from a single null geodesic $\Gamma$ to a set of null geodesics containing $\Gamma$ as a member. There are two noteworthy facts. First, if $\eta^{p}$ is tangent to $\Gamma$ and undergoes parallel transport along it, and if $\delta x^{p}$ is an infinitesimal displacement from $\Gamma$ to a neighbouring null geodesic, then $d J / d \lambda \doteq 0$, where

$$
J=\eta_{D} \delta x^{p}
$$

From the null character of $\eta^{p}$, it follows that the correlation between the points on $\Gamma$ and on its neighbour is immaterial. The second fact is more complicated. $C$ is a point on $\Gamma$ and we take an $\infty^{n-1}$ family of null geodesics passing near $C$ and parallel to $\Gamma$ near $C$. Out of this $\infty^{n-1}$ family we pick the $\infty^{n-2}$ family for which $J$ has some assigned (infinitesimal) value. Let $S_{n-1}$ be a local flat subspace at $C$, and let $S_{n-2}$ be the local flat intersection of $S_{n-1}$ with the $\infty^{n-2}$ family of null geodesics aforesaid. The fact is this : $S_{n-2}$ is perpendicular to the projection of $\Gamma$ on $S_{n-1}$.

With these geometrical preliminaries disposed of, we turn to space-time and consider a star $A$ and an observer $B$ whose two world-lines intersect a null geodesic $\Gamma$ at $C_{1}$ and $C_{0}$ respectively. From the group of special parameters on $\Gamma$ we pick out that one (an additive constant is immaterial) which makes $d \lambda$ agree with the usual definition of spatial distance for objects in $B$ 's infinitesimally small, instantaneous, three-dimensional space. Then (this is the definition, and it is not compared with the definition in $(g)$ ), the spatial distance from $A$ to $B$ is

$$
\Delta=\int_{C_{\mathrm{t}}}^{C_{0}} d \lambda
$$

This is expressed more explicitly as follows. Let $\varpi^{p}$ be the unit tangent vector to $B$ 's world-line at $C_{0}$. Regarding $\Gamma$ as known, but the normalisation of $\lambda$ as unknown, we may write

$$
\frac{d x^{0}}{d \lambda}=k \dot{Y}\left(x^{0}, x^{1}, x^{2}, x^{3}\right)
$$

where $k$ is an unknown constant and $Y$ a known function. Then the formula for spatial distance is

$$
\Delta=Y\left(x_{0}^{0}, x_{0}^{1}, x_{0}^{2}, x_{0}^{3}\right) \frac{\sigma^{p} d x_{0}^{p}}{d x_{0}^{0}} \int_{C_{1}}^{c_{0}} \frac{d x^{0}}{Y\left(x^{0}, x^{1}, x^{2}, x^{3}\right)},
$$

where $d x_{0}^{p}$ refer to $\Gamma$ at $C_{0}$.

This formula is applied to the de Sitter world.

Having thus disposed of the question of spatial distance, the argument moves on to the discussion of wave-fronts. But it gets a little muddy, and does not reveal clearly just what a wave-front is. Undoubtedly, the history 


\section{WHITTAKER'S CONTRIBUTIONS TO RELATIVITY}

of a wave-front is a family of geodesics, but what sort of family? The clear and simple answer was intrinsic in the paper $(d)$, written five years earlier; surely the history of a wave-front is nothing but a characteristic 3 -space, a solution of (2) of $(d)$ above, a 3-space formed of null geodesics and tangent at each of its points to the elementary null-cone? Doubtless the discussion given in the paper leads to the same conclusion, but a point is missed that might have been made.

To treat the Doppler effect in terms of wave-fronts, we go back to the star $A$ and the observer $B$, mentioned above, and consider two adjacent wave-fronts travelling from $A$ to $B$. Each contains a null geodesic joining the world-lines of the star and the observer. Let $\eta^{p}$ be a transport vector, with values ${ }_{1} \eta^{p}$ at $A$ and ${ }_{0} \eta^{p}$ at $B$, and let $\rho_{p}$ and $\varpi_{p}$ be unit covariant vectors along the world-lines of $A$ and $B$ respectively. The two wave-fronts determine wave lengths, $\lambda_{1}$ at $A$ and $\lambda_{0}$ at $B$. By means of the invariant $J$ of (2), the following formula is obtained for the Doppler effect :

$$
\frac{\lambda_{0}}{\lambda_{1}}=\frac{{ }_{1} \eta^{p} \rho_{p}}{{ }_{0}^{p} \varpi_{p}}
$$

This formula is applied to flat space-time and to the de Sitter world.

Finally, the transport vector $\eta^{p}$ is identified with the momentum-energy vector of a photon traversing the null geodesic, and the theory is linked to quantum ideas by the formula $E=h \nu$, which effectively assigns a wave-length to the photon. Hence it is shown that Doppler effects in general relativity are the same, whether calculated from the wave theory of light or from the energy of the associated light-quanta. Applications are made to the statical universe and to the expanding universe.

(i) "On Gauss' theorem and the concept of mass in general relativity," Proc. Roy. Soc. A, 149 (1935), 384-395.

The classical theorem of Gauss is expressed by the formula

$$
\iint \frac{\partial V}{\partial v} d S=4 \pi M
$$

where $V$ denotes the gravitational potential, $S$ a simple closed surface of which $d S$ is the element of area, $d \nu$ the element of inward-drawn normal to $d S$, and $M$ the total gravitating mass contained within the surface $S$. This theorem is to be generalized from Newtonian physics to general relativity. The argument is, however, restricted to those universes with metric of the form

$$
d \tau^{2}=U d t^{2}-c^{-2} a_{p q} d x^{p} d x^{q}
$$

where $a_{p q}$ depend only on the coordinates $x^{1}, x^{2}, x^{3}$ (Latin suffixes run $1,2,3$ ).

The gravitational force on a particle (or observer) pursuing a world-line with equations $x^{a}=x^{a}(\dot{\tau})$ (Greek suffixes run $0,1,2,3$ ) is defined as the fourvector

$$
g^{\alpha}=-\left[\frac{d^{2} x^{a}}{d \tau^{2}}+\left\{\begin{array}{c}
a \\
\beta \gamma
\end{array}\right\} \frac{d x^{\beta}}{d \tau} \frac{d x^{\gamma}}{d \tau}\right]
$$


When calculated for a particle at rest, so that $d x^{i} / d \tau=0$, this gives

$$
g^{i}=-\frac{1}{2} c^{2} \frac{a^{i k}}{U} \frac{\partial U}{\partial x^{k}}, \quad g^{0}=0
$$

Whittaker considers, as the appropriate generalization of the left-hand side of (1), the integral

$$
I=-c^{3} \iint\left\{g^{1} \frac{\partial\left(x^{2}, x^{3}\right)}{\partial(u, v)}+g^{2} \frac{\partial\left(x^{3}, x^{1}\right)}{\partial(u, v)}+g^{3} \frac{\partial\left(x^{1}, x^{2}\right)}{\partial(u, v)}\right\} \sqrt{-g} d u d v
$$

taken over any simple closed surface $S$ in the space of $\left(x^{1}, x^{2}, x^{3}\right)$, on which $u$ and $v$ are parameters ; $g$ is the determinant of the coefficients in (2), so that

$$
\sqrt{-g}=c^{-3} U^{\mathbf{t}} a^{\mathbf{l}}, \quad a=\operatorname{det} a_{p q}
$$

The constant factor $-c^{3}$ in (5) is suggested by calculating $I$ over a sphere $r=$ const. in the Schwarzschild field (4) of (c); with $a=2 \beta M / c^{2}$, where $\beta$ is the Newtonian constant of gravitation, the result is $I=4 \pi \beta M$, so that the righthand side of $(1)$ is essentially reproduced.

The next step is to change $I$ to a volume-integral, and use the field equations to express the integrand in terms of the energy-tensor of matter. The volumeintegral is

$$
I=c^{2} \iiint \Delta_{2} U ! \cdot a^{\frac{1}{1}} d x^{1} d x^{2} d x^{3},
$$

where the operator $\Delta_{2}$ is Beltrami's differential parameter of the second order, such that

$$
\Delta_{2} V=a^{-1} \frac{\partial}{\partial x^{h}}\left(a^{\frac{1}{2}} \cdot a^{h k} \frac{\partial V}{\partial x^{k}}\right)
$$

Now the Ricci tensor for the metric (2) has the mixed component

$$
K_{0}^{0}=-c^{2} U^{-1} \Delta_{2} U^{t}
$$

and so, making use of the field equations of gravitation, we get

$$
I=\frac{8 \pi \beta}{c^{2}} \iiint\left(T_{0}^{0}-\frac{1}{2} T\right) \sqrt{-g} d x^{1} d x^{2} d x^{3},
$$

where $T_{\beta}^{a}$ is the energy-tensor. This formula, which equates the surfaceintegral $I$ of (5) to a volume-integral involving the energy-tensor of matter, is the generalization of Gauss' theorem in Newtonian potential theory.

But of course the energy-tensor may not be due to matter-it may be due to an electrostatic field. Whittaker considers a condenser with an electrostatic field in it with energy-tensor $E_{\beta}^{a}$. But mechanical stresses are required to hold the surface-elements of the condenser in equilibrium, and so he adds a stress-system $R_{q}^{p}$, with the result that only the component $T_{0}^{0}$ survives in the total energy-tensor $T_{\beta}^{a}=E_{\beta}^{a}+R_{\beta}^{a}$. Then $T=T_{0}^{0}$, and (10) gives $I=4 \pi \beta M$, where

$$
M c^{2}=\iiint T_{0}^{0} \sqrt{-g} d x^{1} d x^{2} d x^{3}
$$


the total energy of the system. I confess I cannot follow this argument, for I cannot see any energy-tensor but that of the field in the space between the walls of the condenser, and not including them.

The field (2) is now specialized by making $U$ independent of $t$, and Whittaker is led to define the potential mass of a moving particle as

$$
m\left(1-\frac{w^{2}}{c^{2}}\right)^{-\frac{1}{2}}
$$

where $w$ is the speed the particle would have if it got to infinity, where $U=1$. The quantity $w^{2}$ may be negative-it is negative if the particle has not sufficient energy to carry it out of the gravitational field into the Galilean field at infinity. This idea of potential mass is used to state another theorem involving the integral $I$ of $(5)$ to the effect that when the statical gravitational field is due solely to material particles, then $I=4 \pi \beta M$, the integral being taken over any simple closed surface in the space of $\left(x^{1}, x^{2}, x^{3}\right)$ and $M$ being the sum of the potential masses of those particles that are inside this surface.

This is a striking result, but not quite as good as it seems at first sight, for (to get a statical field) the particles must be at rest, and, in order that they may remain at rest, they must be situated at places for which $U$ has maximum or minimum values, as is remarked in a footnote.

The paper ends with the extension to general relativity of Gauss' theorem in its electrostatic form. Let $S$ be a simple closed surface (2-space) which is the frontier of a three-dimensional domain (or multipoint) $M$. Then the total quantity of electricity belonging to those world-lines of electric charge which interesect $M$ is expressible as a triple integral over $M$, the integrand involving the 4-current. This integral can be transformed into a double integral over $S$, use being made of Maxwell's equations, and the result is that the total charge in question is equal to the following integral over $S$, in which $X^{\alpha \beta}$ is the electromagnetic tensor, or six-vector :

$$
\begin{aligned}
& \iint\left\{X^{23} \frac{\partial\left(x^{0}, x^{1}\right)}{\partial(u, v)}+X^{31} \frac{\partial\left(x^{0}, x^{2}\right)}{\partial(u, v)}+X^{12} \frac{\partial\left(x^{0}, x^{3}\right)}{\partial(u, v)}\right. \\
& \left.\quad+X^{03} \frac{\partial\left(x^{1}, x^{2}\right)}{\partial(u, v)}+X^{02} \frac{\partial\left(x^{3}, x^{1}\right)}{\partial(u, v)}+X^{01} \frac{\partial\left(x^{2}, x^{3}\right)}{\partial(u, v)}\right\} \sqrt{-g} d u d v .
\end{aligned}
$$

(j) "On the relations of the tensor-calculus to the spinor-calculus," Proc. Roy. Soc. A, 158 (1937), 38-46.

Although there is a rather vague indication of its use in general relativity, the argument deals essentially with flat space-time with Galilean coordinates $x^{r}(r=0,1,2,3)$, so that the metric tensor is the diagonal matrix $g_{m n}=(1,-1$, $-1,-1)$. Against this background we see, on the one hand, tensors which transform under Lorentz transformations according to the well-known rules, and, on the other hand, spinors consisting each of a pair of complex numbers $\left(\phi_{1}, \phi_{2}\right)$ transforming according to the unimodular transformation

$$
\phi_{1}^{\prime}=a \phi_{1}+\beta \phi_{2}, \quad \phi_{2}^{\prime}=\gamma \phi_{1}+\delta \phi_{2}
$$

E.M.S.-D 2 
where $a \delta-\beta \gamma=1$. The link between tensors and spinors rests on the fact that any spin-transformation (1) corresponds to a Lorentz transformation, but Whittaker's purpose is to make the linkage more concrete by constructing what might be called a tensorial model of a spinor. This model is a pair of complex numbers constructed out of the components of a complex self-dual skew-symmetric tensor with vanishing invariant.

The treatment of dual tensors is made much clearer if we insert the permutation symbol $\epsilon_{p q r s}$ into Whittaker's work. Under proper Lorentz transformations it is a covariant tensor. If we raise the suffixes by means of $g^{m n}$, we get the contravariant tensor $\epsilon^{p q r s}\left(=-\epsilon_{p q r s}\right)$. The relationship between a (complex) skew-symmetric tensor $X_{p q}$ and its dual $Y_{p q}$ can be expressed in several equivalent forms, such as

$$
Y_{p q}=\frac{1}{2} i \epsilon_{p q r s} X^{r s}, X^{p q}=\frac{1}{2} i \epsilon^{p q r s} Y_{r s} .
$$

A tensor $R_{p q}$ is self-dual if it satisfies the (Lorentz-invariant) condition

or equivalently

$$
R_{p q}=\frac{1}{2} i \epsilon_{p q r s} R^{r s} \text {, }
$$

$$
R_{01}=i R_{23}, R_{02}=i R_{31}, R_{03}=i R_{12} \text {. }
$$

Any skew-symmetric tensor possesses two invariants, of which one is $R_{p q} R^{p q}$, and we may say that it is $n u l l$ if both these invariants vanish. However, if the tensor is self-dual, one condition suffices to make it null, and that condition is

$$
R_{01}^{2}+R_{02}^{2}+R_{03}^{2}=0 \text {. }
$$

We are concerned with tensors which satisfy (4) and (5). Remembering that the tensor has complex components, we recognize that it has four real degrees of freedom, the same number as for a spinor.

The spin-transformation (1) corresponds to a Lorentz transformation with coefficients formed quadratically from $\alpha, \beta, \gamma, \delta$ according to a certain well. known plan. Thus we can write down tensorial formulæ of transformation for $R_{p q}$. We might expect the coefficients in the transformation of this secondorder tensor to be of the fourth degree in $\alpha, \beta, \gamma, \delta$, but (on account of the unimodular condition) they turn out to be quadratic. Combining these formulæ of transformation suitably, we find that

$$
\begin{aligned}
& R_{01}^{\prime}+i R_{02}^{\prime}=\alpha^{2}\left(R_{01}+i R_{02}\right)+\beta^{2}\left(-R_{01}+i R_{02}\right)-2 \alpha \beta R_{03} \\
& -R_{01}^{\prime}+i R_{02}^{\prime}=\gamma^{2}\left(R_{01}+i R_{02}\right)+\delta^{2}\left(-R_{01}+i R_{02}\right)-2 \gamma \delta R_{03} .
\end{aligned}
$$

Here comes the dramatic touch : these equations are the squares of the equations

$$
\begin{aligned}
& \left(R_{01}^{\prime}+i R_{02}^{\prime}\right)^{\frac{1}{2}}=\alpha\left(R_{01}+i R_{02}\right)^{\frac{1}{2}}+\beta\left(-R_{01}+i R_{02}\right)^{\frac{1}{2}}, \\
& \left(-R_{01}^{\prime}+i R_{02}^{\prime}\right)^{\frac{1}{2}}=\gamma\left(R_{01}+i R_{02}\right)^{\frac{1}{2}}+\delta\left(-R_{01}+i R_{02}\right)^{\frac{1}{2}},
\end{aligned}
$$

and these last equations are precisely the equations of the spin-transformation (1) corresponding to the Lorentz transformation we have been using.

Introducing a convenient factor $\sqrt{2}$, Whittaker is able to state that, if $R_{p q}$ is self-dual and null, then

$$
\sqrt{2} \phi_{1}=\left(R_{01}+i R_{02}\right)^{\frac{1}{2}}, \quad \sqrt{2} \phi_{2}=\left(-R_{01}+i R_{02}\right)^{\frac{1}{2}}
$$


are the components of a spinor; expressing the tensor in terms of the spinor he writes

$$
\begin{aligned}
& R_{01}=\phi_{1}^{2}-\phi_{2}^{2}, R_{02}=-i\left(\phi_{1}^{2}+\phi_{2}^{2}\right), R_{03}=-2 \phi_{1} \phi_{2}, \\
& R_{23}=-i\left(\phi_{1}^{2}-\phi_{2}^{2}\right), R_{31}=-\left(\phi_{1}^{2}+\phi_{2}^{2}\right), R_{12}=2 i \phi_{1} \phi_{2} .
\end{aligned}
$$

This is the central point. The desired connection has been set up between a spinor and a tensor. The rest of the paper is devoted to applications of this idea.

If $\left(\phi_{1}, \phi_{2}\right)$ and $\left(\psi_{1}, \psi_{2}\right)$ are two spinors, it is known from spinor-calculus that we can form a vector $D^{p}$ out of them by writing (the star indicates the complex conjugate)

$$
\begin{aligned}
& D^{\mathbf{0}}=\phi_{1} \psi_{1}^{*}+\phi_{2} \psi_{2}^{*}, D^{1}=\phi_{1} \psi_{2}^{*}+\psi_{1}^{*} \phi_{2}, \\
& D^{2}=i \phi_{1} \psi_{2}^{*}-i \psi_{1}^{*} \phi_{2}, D^{3}=\phi_{1} \psi_{1}^{*}-\phi_{2} \psi_{2}^{*} .
\end{aligned}
$$

How is $D^{p}$ connected with the two self-dual null tensors, say $R_{p q}$ and $S_{p q}$, which correspond to the two spinors? The answer is as follows :

$$
R_{\cdot k}^{p \cdot} S^{* q k}=-2 D^{p} D^{q} .
$$

Another application : if $H_{p}$ is the vector

$$
H_{p}=\phi_{2} \frac{\partial \phi_{1}}{\partial x^{p}}-\phi_{1} \frac{\partial \phi_{2}}{\partial x^{p}}
$$

defined by the single spinor $\left(\phi_{1}, \phi_{2}\right)$, and $R_{p q}$ is the tensor corresponding to this spinor, then

$$
R_{k s} D_{\mid p}^{k}=2 D_{s} H_{p}
$$

where $D^{p}$ is the vector as in (10), formed by introducing a second arbitrary "catalytic" spinor $\left(\psi_{1}, \psi_{2}\right)$.

Finally, Whittaker translates Dirac's equations for the electron into a vector equation. Starting from Weyl's form of Dirac's equations, in which the two spinors are $\left(\psi_{1}^{*}, \psi_{2}^{*}\right)$ and $\left(\psi_{4},-\psi_{3}\right)$, the steps are as follows.

First, self-dual null tensors $M_{p q}^{*}$ and $R_{p q}$ are formed out of the two spinors, as in (9). Next, vectors $A^{p}, B^{p}$ and $C^{p}$ are defined by

$$
M_{p k} M^{* q k}=-2 A_{p} A^{q}, R_{p k} R^{* q k}=-2 B_{p} B^{q}, M_{p k} R^{q k}=-2 C_{p} C^{q} .
$$

Next, vectors $P_{p}$ and $Q_{p}$ are defined by

$$
M_{k s} A_{\mid p}^{k}=2 A_{s} P_{p}, R_{k \varepsilon}^{*} B_{\mid p}^{k}=2 B_{s} Q_{p}
$$

Finally, vectors $S_{p}$ and $T_{p}$ are defined by

$$
S_{p}=\left(M_{p}^{\cdot q}\right)_{1 q}, T_{p}=\left(R_{p}^{* . q}\right)_{1 q} .
$$

Then Dirac's equations are equivalent to $\Omega_{p}=0$, where

$$
\Omega_{p}=\frac{1}{2} S_{p}+\frac{1}{2} T_{p}+P_{p}+Q_{p}+\frac{m c i}{\hbar}\left(C_{p}+C_{p}^{*}\right) \text {. }
$$

Dublin Institute for Advanced Studies

Merrion Square

DUBLIN

IRELAND 